P. DAINESI

L. THEVENAZ

PH. ROBERT

\title{
Intensity modulation in two Mach-Zehnder interferometers using plasma dispersion in silicon-on-insulator
}

\author{
EPFL, Swiss Federal Institute of Technology, Metrology Laboratory, 1015 Lausanne, Switzerland
}

\begin{abstract}
Received: 18 May 2001/Revised version: 24 September2001 Published online: 30 October 2001 • (C) Springer-Verlag 2001

ABSTRACT We present comparative measurements of two Mach-Zehnder interferometers, one with $Y$-junction couplers and the other with MMI couplers, both developed in siliconon-insulator technology and using plasma dispersion effect for light phase modulation. Measurements of fiber-to-fiber losses, absorption coefficient, output intensity vs. time and extinction ratio vs. frequency have been performed at $\lambda=1.3 \mu \mathrm{m}$ and at $\lambda=1.55 \mu \mathrm{m}$. Results are reported and discussed in this paper.
\end{abstract}

PACS 42.25.Hz; $42.82 \mathrm{~Bq} ; 42.82 \mathrm{Et} ; 42.82 \mathrm{Gw} ; 42.79 \mathrm{Hp}$

1

Introduction

Mach-Zehnder interferometers (MZI) in siliconon-insulator (SOI) technology have been and still are the subject of much interest due to the high potential of silicon optoelectronic devices. Silicon is highly transparent in the infrared spectral region and its electronic capabilities are well known, making SOI a very attractive technology combining optical and electronic functions with optimal features on the same substrate.

MZIs using standard $Y$-junction couplers based on the thermo-optic effect have already been proposed in the past. These devices have a limited extinction ratio of about $13 \mathrm{~dB}$ for the best reported [1,2,4], and are bandwidth-limited to about $100 \mathrm{kHz}[1,2]$, except for a special device operating up to $700 \mathrm{kHz}$ [5]. Multimode interference (MMI) couplers in SOI technology have been proposed for the first time in thermo-optic $1 \times 2$ and $2 \times 2$ switches [3].

MZIs using standard $Y$-junction couplers based on the plasma dispersion effect have also been proposed in the past $[6,7]$. A response time of $50 \mathrm{~ns}$ and a modulation depth of $-4.9 \mathrm{~dB}$ are reported in [6], while a modulation depth of $98 \%$ measured in [7].

We present two MZIs, one with $Y$-junction-couplers and one with MMI couplers, both based on the plasma dispersion effect. A $Y$-junction-couplers MZI based on the thermo-optic effect has also been realized on the same substrate for performance comparison. The fiber-to-fiber insertion losses, ab-

Fax: +41-21/693 26 14, E-mail: paolo.dainesi@epfl.ch sorption coefficients, optical coefficients modulated intensities as functions of time, and modulation extinction ratios versus signal frequencies for these devices were measured at the wavelengths $1.3 \mu \mathrm{m}$ and $1.55 \mu \mathrm{m}$. At $1.3 \mu \mathrm{m}$, polarizationsensitive extinction ratio measurements were also performed. Comparative results are reported and discussed in this paper. The $Y$-junction MZI shows the best extinction ratio reported to date, and the MMI MZI is the first device presented in this configuration.

\section{Devices description}

The devices were realized by etching rib waveguides in a separation by implanted oxygen (SIMOX) SOI wafer, the cross-section being shown in Fig. 1a. SOI wafers were originally developed to solve some of the problems shown in bulk silicon electronics related to the stray capacitance between the doped regions and the substrate. The SIMOX process consists basically of two steps [12]: first, a heavy dose of oxygen is implanted on a standard silicon substrate. Actually the reference parameters are: oxygen dose of $1.8 \times 10^{18} \mathrm{~cm}^{-2}$ at $200 \mathrm{keV}$ and a temperature above $500^{\circ} \mathrm{C}$. Second, a thermal annealing (ideally $6 \mathrm{~h}$ in a $2 \%$ oxygennitrogen ambient at a temperature of $1300-1500^{\circ} \mathrm{C}$ ) is performed to obtain a $\mathrm{SiO}_{2}$ layer with a thickness $t=400 \mathrm{~nm}$ beneath a Si overlayer with a thickness of about 140-150 nm. In this thin layer dislocation defects due to the oxygen implant process can be very high, and to obtain optically transparent wafers a successive epitaxial growth of silicon is performed to attain the final height $H$ (Fig. 1a).

On these wafers, a rib can be etched to form an optical waveguide. To do this we performed a non-pulsed room temperature dry etching with $\mathrm{SF}_{6} / \mathrm{C}_{4} \mathrm{~F}_{8}$ gas mixtures. Vertical sidewalls with small residual roughness were obtained with a very well controlled depth.

Large ribs also make possible low loss couplings to optical fibers (theoretically down to $0.35 \mathrm{~dB} /$ facet) as a result of the very good fiber-waveguide fundamental modes overlapping. Single mode propagation is also achieved when the well-known [10] geometrical condition $W / H \leq 0.3+$ $\left(1-r^{2}\right)^{-1 / 2}$ is fulfilled, where $r$ is the etching ratio (Fig. 1a). For all our devices we have $H=W=10 \mu \mathrm{m}, r=0.6$. Figure $1 \mathrm{~b}$ and $1 \mathrm{c}$ show a top-view of the two proposed MZIs. The MMI couplers in Fig. $1 \mathrm{~b}$ were designed to achieve a $3 \mathrm{~dB}$ 

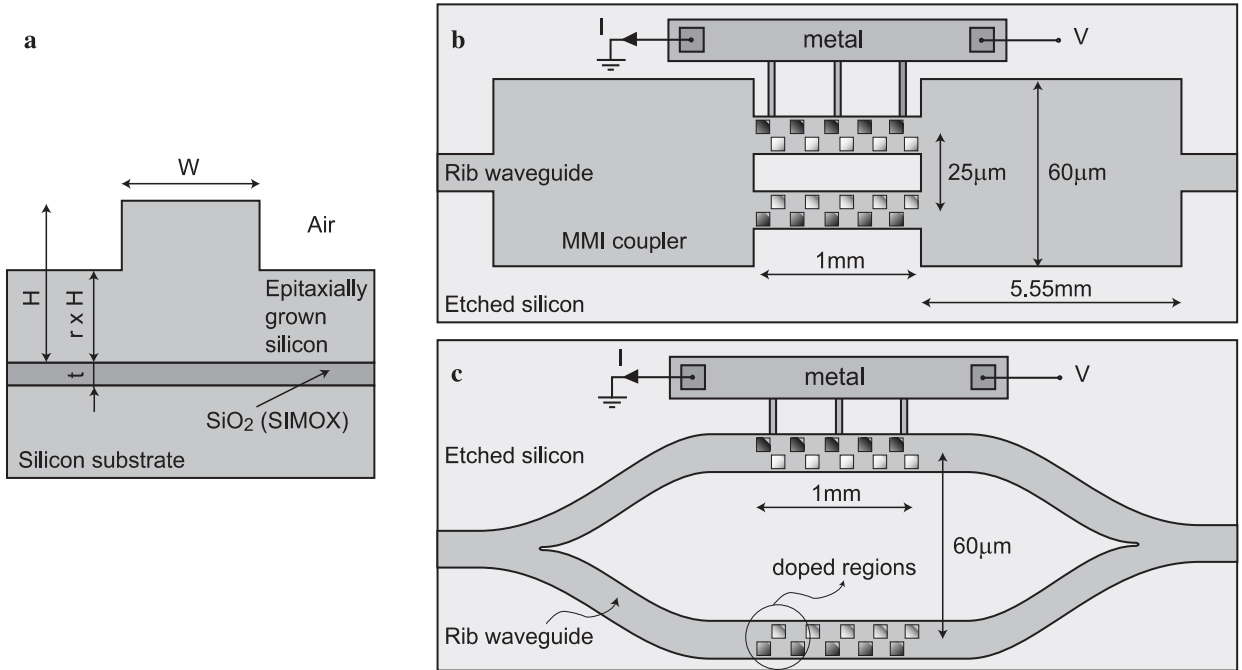

FIGURE 1 Schematic description of the intensity modulators: a rib waveguide cross-section; b MZI with 3-dB MMI couplers; c MZI with $Y$-junctioncouplers

splitting ratio at $\lambda=1.3 \mu \mathrm{m}$ using the restricted multimode interference method [8] for symmetric interference. Assuming the MMI input waveguide is at the center and only the fundamental mode is present to excite multimode propagation in the MMI coupler input, $N$-fold images of the input field are self-imaged at a distance $L=p / N\left(3 L_{\pi} / 4\right)$ [8], where $p$ is an integer greater than 0 and $L_{\pi}$ is the beat length between the two lowest-order modes.

To modulate the phase of the light propagating through one arm of these MZIs we have implemented electronic diodes on top of a waveguide segment designed to be $100 \%$ CMOS compatible. Doped regions, metal connections and pads are sketched in Fig. $1 \mathrm{~b}$ and 1c, while a detailed image of the diodes' integration technology is presented, for a small

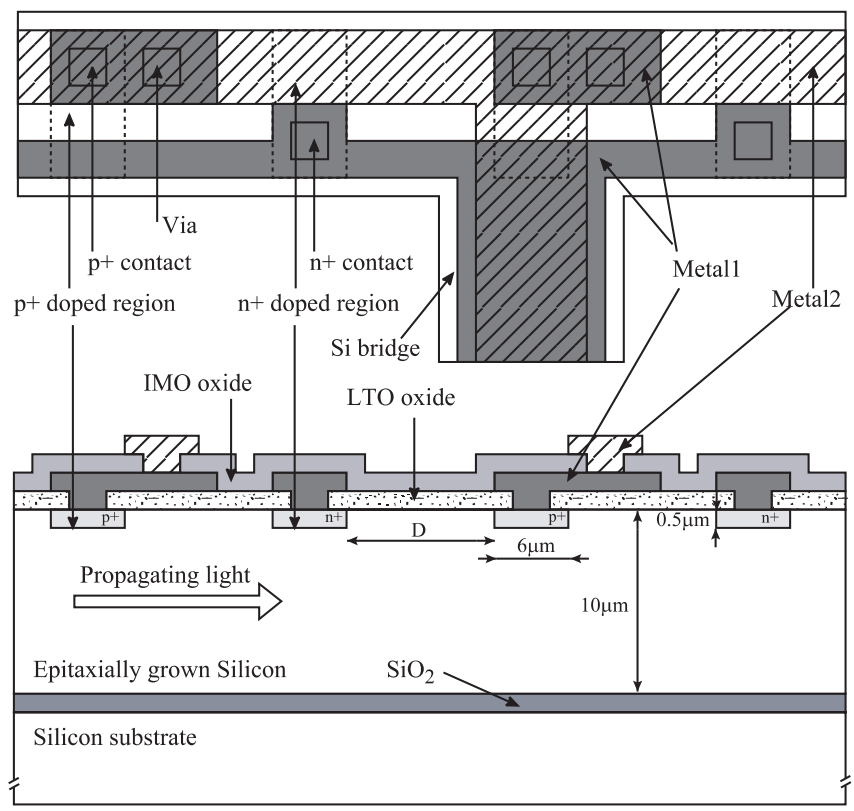

FIGURE 2 Detailed image of the integration design of the diodes responsible for light phase modulation. Doped regions, contacts, vias and metals are evidenced in a portion of the waveguide waveguide portion, in Fig. 2. A portion of the silicon bridge, which connects metal stripes to the diodes on the waveguide, is also visible in Fig. 2. The distance between the doped regions forming the diodes ( $D$ in Fig. 2 ) has been made quasiperiodic in order to avoid possible unwanted multiple reflection of the propagating mode. The diode injected charge is responsible for light phase modulation through the plasma dispersion effect, as stated in [6] among others.

\section{$3 \quad$ Measurement results and discussion}

First we performed fiber-to-fiber insertion loss measurements for both devices. The light sources were multimode laser diodes at $\lambda=1.3 \mu \mathrm{m}$ and at $\lambda=1.55 \mu \mathrm{m}$ to prevent any Fabry-Perot interference in the device. A polarizing fiber and an all-fiber polarization controller enabled us to perform polarization-sensitive measurements; a crosstalk better than $25 \mathrm{~dB}$ between the TE and TM modes of the incoming light has been measured. For $V=I=0$ (see Fig. 1) no charge is injected and the MZIs are in their ON state; we measured the fiber-to-fiber insertion losses as the ratio between the output and input powers. The results at $\lambda=1.3 \mu \mathrm{m}$ are $9.5 \mathrm{~dB}$ for the $Y$-junction MZI and $9.4 \mathrm{~dB}$ for the MMI MZI, while at $\lambda=1.55 \mu \mathrm{m}$ they are $9.6 \mathrm{~dB}$ and $11.5 \mathrm{~dB}$, respectively. The higher insertion loss of the MZI with MMI couplers at $1.55 \mu \mathrm{m}$ is due to the fact that the MMI coupler is designed to be $3 \mathrm{~dB}$ at $1.3 \mu \mathrm{m}$, which is a little too long for correct operation at $1.55 \mu \mathrm{m}$, as the beat length $L_{\pi}$ at $1.55 \mu \mathrm{m}$ is shorter. The fiber-to-fiber insertion loss of a reference simple straight waveguide was also measured to be $7.2 \mathrm{~dB}$ for both wavelengths, showing the fairly low excess loss $(<2.3 \mathrm{~dB}$ at $1.3 \mu \mathrm{m})$ of the two MZIs. To better evaluate the losses in these devices we performed a Fabry-Perot interference measurement of the absorption coefficient in the same reference straight waveguide. To do this we modulated over about $6 \mathrm{GHz}$ the output frequency of two DFB lasers, one at $\lambda=1.31 \mu \mathrm{m}$ and the other at $\lambda=1.557 \mu \mathrm{m}$. By measuring the waveguide cavity contrast as the ratio between the maximum and the minimum value in the resulting transmission 
interference fringes, the absorption coefficient can be determined using the well-known relation reported in [11], that was derived directly from the Fabry-Perot interference transmission expression. We measured at both wavelengths a cavity contrast of 1.9 and then for a waveguide length of $2 \mathrm{~cm}$ and a reflectivity (air-silicon) on both facets of 0.31 , we measured $\alpha=1.4 \mathrm{~dB} / \mathrm{cm}$, in agreement with [9]. Using this result, together with the result of the fiber-to-fiber loss, we can then estimate the value of the fiber-waveguide coupling efficiency. In the measured 7.2-dB fiber-to-fiber loss, the measured absorption loss is $2.8 \mathrm{~dB}$ and the reflection loss is $3.2 \mathrm{~dB}$ for both facets, estimated with known values of the index of refraction. The remaining $1.2 \mathrm{~dB}$ loss is the injection loss on both facets. The resulting fiber-waveguide coupling efficiency is then $\eta=0.87$, which is close to the theoretical value and is quite remarkable for a fiber-waveguide end-butt coupling with no mode-matching optics.

We then applied a modulating square current signal $I=$ $150 \mathrm{~mA}$, and measured the output intensity of both devices as a function of time. The applied powers, determined by measurement of the voltage drop across the diodes, were $330 \mathrm{~mW}$ and $360 \mathrm{~mW}$ for the $Y$-junction MZI and the MMI MZI, respectively. Results for a $20-\mathrm{kHz}$ signal frequency at $\lambda=1.3 \mu \mathrm{m}$ are shown in Fig. 3. The intensities are normalized relative to the $\mathrm{ON}$ level (the output intensity for $I=0$ ). The OFF level corresponds to no injected light (average dark current of the measuring output photodiode). In Fig. 3 we clearly see the counter-modulation due to the thermo-optic effect after the charge injection, which prevents the signal staying in the OFF state. It is important to notice that unless one has very efficient diodes capable of injecting high current levels for reasonably low voltage drops, the thermo-optic effect will be a major drawback in charge injection devices at low frequencies. In particular, in Fig. 3 it is possible to see the performance difference between the two devices showing different thermo-optic-effect drawbacks (5\% for the $Y$-junction MZI and about 20\% for the MMI coupler MZI). This difference between the two devices can be partially explained by the different dissipated powers, but it is mainly due to the

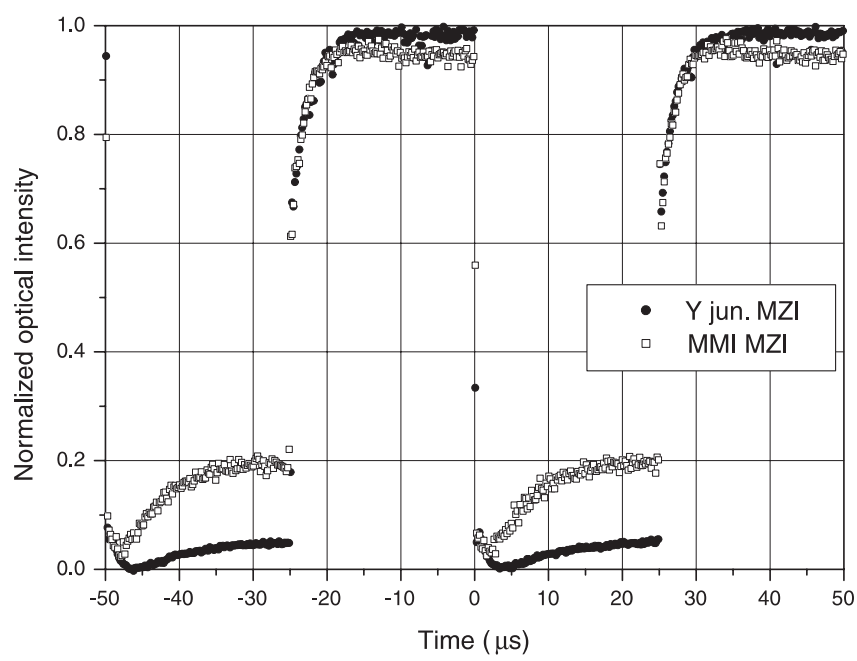

FIGURE 3 Normalized optical intensity as a function of time for the two Mach-Zehnder interferometers for a modulating signal frequency of $20 \mathrm{kHz}$. Thermo-optic counter-modulation can be observed different geometry. It should be pointed out that charge injection is always associated with thermal dissipation and a phase modulator purely based on the plasma dispersion effect cannot be realized. But for a high modulating signal frequency (over $200 \mathrm{kHz}$ for our experiment), the thermo-optic-effect becomes a simple temperature offset, changing the dc operating point. This change can then be corrected by adjusting the dc injected current. Results clearly showing this effect, obtained for a $1 \mathrm{MHz}$ signal frequency at $\lambda=1.55 \mu \mathrm{m}$, are shown in Fig. 4. The thermo-optic effect has averaged out and the modulating signal dc value has been adjusted to compensate for the thermal offset. Here the most important result is the differing abilities to reach the OFF level shown by the two devices. The MMI MZI is limited to about $4 \%$ of the maximum value while the $Y$-junction MZI can reach $1 \%$ of the maximum value at this modulating-signal frequency. This apparently small difference results in a 6-dB difference on an extinction ratio logarithmic scale. The fact that the MMI is not working properly as a $3-\mathrm{dB}$ coupler could reasonably explain the observed difference in performance. We can also assume that mutual coupling between modes propagating in the two arms at the output of the MMI coupler can be induced as a result of the relatively small distance between the arms themselves. In order to reduce this mutual coupling we assume thinner waveguides would be a good solution $(H=3 \mu \mathrm{m}$, for example). Actually, in thinner waveguides, for an identical MMI width and wavelength, a greater number of modes can propagate in the MMI coupler, resulting in a better resolution of the input field self-imaging and, consequently, in a better crosstalk of the two output fields. The 10\%-90\% rise (OFF switching) and fall (ON switching) time for the modulated signals in Fig. 4 is about $100 \mathrm{~ns}$, in good agreement with [6]. We can thus estimate the bandwidth of the plasma dispersion effect for these devices to about $3.5 \mathrm{MHz}$.

In Fig. 5 a measurement of the extinction ratio as a function of the modulating signal frequency at $\lambda=1.3 \mu \mathrm{m}$ is shown. The measurement set-up dynamic range is $22 \mathrm{~dB}$.

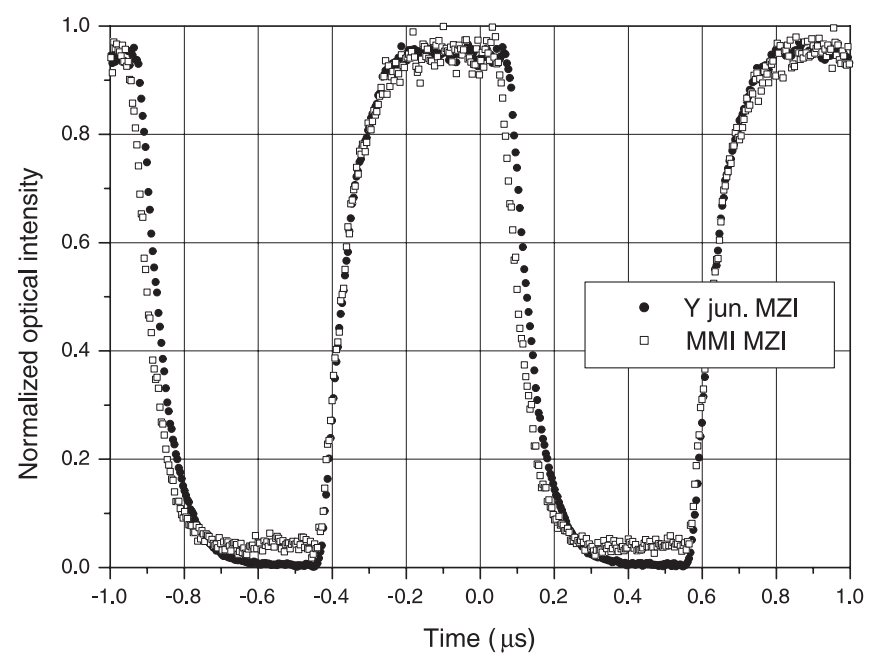

FIGURE 4 Normalized optical intensity as a function of time for the two Mach-Zehnder interferometers for a modulating signal frequency of $1 \mathrm{MHz}$. The thermo-optic effect is now a thermal offset that has been corrected by adjusting the dc operating point of the charge injecting diodes. The plasma dispersion effect bandwidth can also be estimated 


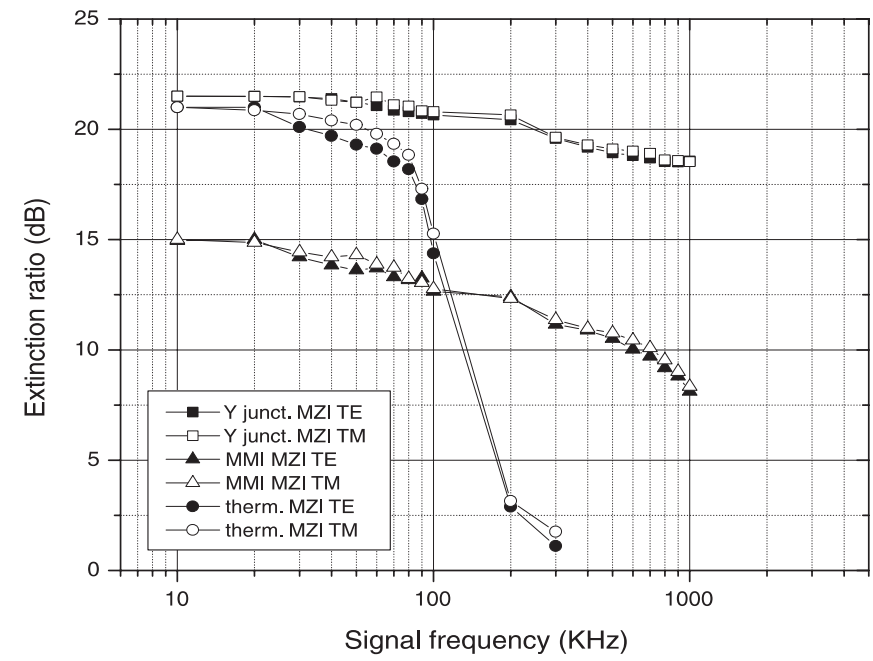

FIGURE 5 Extinction ratio as a function of the modulating signal frequency of the two devices at $\lambda=1.3 \mu \mathrm{m}$. A bandwidth comparison with a standard thermo-optical MZI is also shown as well as the polarization independence

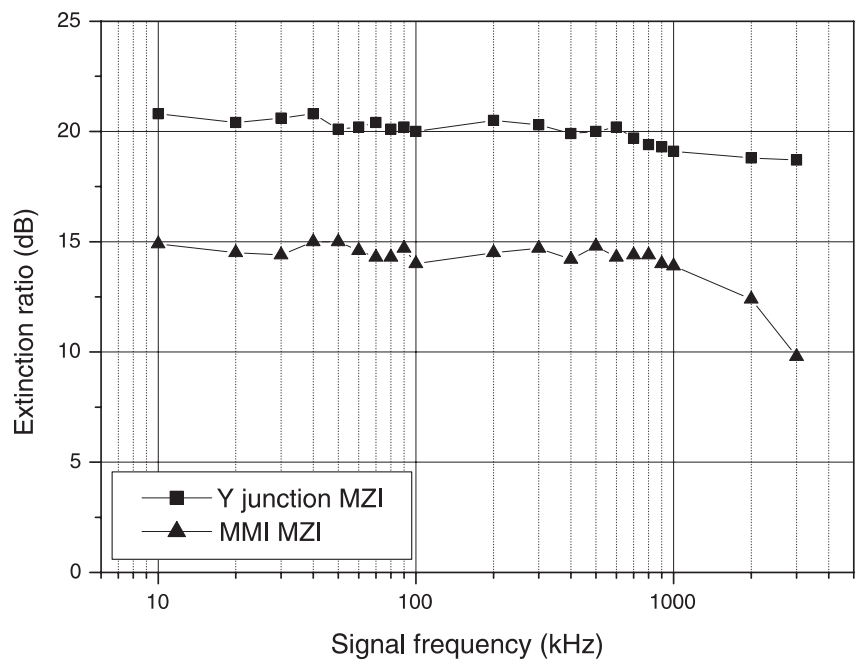

FIGURE 6 Extinction ratio as a function of the modulating signal frequency of the two devices at $\lambda=1.55 \mu \mathrm{m}$. The adjustment of the dc injected current allows unvaried performance up to $1 \mathrm{MHz}$

In this measurement a difference of almost $6 \mathrm{~dB}$, as already mentioned, is systematically observed between the extinction ratios of the $Y$-junction MZI and of the MMI-couplers MZI. This difference places the two devices in distinct application categories. Nevertheless, we think that a certain amount of improvement is possible concerning the MMI-couplers MZI using thinner waveguides, as proposed above.

In order to compare these results in terms of bandwidth, the thermo-optical $Y$-junction MZI, mentioned at the beginning, has been measured also, and its extinction ratio is also reported in Fig. 5. This shows clearly the difference between the thermo-optic effect and the plasma dispersion effect in terms of bandwidth.

Light-polarization independence was also demonstrated (see Fig. 5) using alternatively TE and TM launching at the modulator input.

Measurements of the extinction ratio as a function of the modulating signal frequency have been performed also at $\lambda=1.55 \mu \mathrm{m}$ and the results are shown in Fig. 6. In this last case we could perform the adjustment of the dc injected current to correct the thermal offset at high frequency. This correction could not be performed in our previous measurement at $\lambda=1.3 \mu \mathrm{m}$ due to the current limitation of the signal source. This explains the extinction ratio drop at $\lambda=1.3 \mu \mathrm{m}$, visible in Fig. 5, starting from about $300 \mathrm{kHz}$.

\section{4}

\section{Conclusion}

We have designed and realized a $Y$-junction MZI in SOI based on the plasma dispersion effect which shows the best performances, to our knowledge, in terms of extinction ratio reported to date. We also reported results for the first MMI-couplers MZI using the plasma dispersion effect in SOI. Both devices can be modulated up to a frequency of $3.5 \mathrm{MHz}$. Insertion losses at $\lambda=1.3 \mu \mathrm{m}$ are less than $9.5 \mathrm{~dB}$ and excess losses relative to a straight waveguide in the same technology are less than $2.3 \mathrm{~dB}$. One of the key advantages of our proposed design is the possibility that an optoelectronic functionality can be added to any electronic circuit with a simple single mask postprocessing stage added to the standard CMOS integration process.

\section{REFERENCES}

1 U. Fischer, T. Zinke, B. Schüppert, K. Petermann: Electron. Lett. 30, 406 (1994)

2 G.V. Treyz: Electron. Lett. 27, 118 (1991)

3 U. Fischer, T. Zinke, K. Petermann: IEEE Proc. Int. SOI Conf. (1995) pp. 141-142

4 G. Cocorullo, I. Rendina: Electron. Lett. 28, 83 (1992)

5 G. Cocorullo, M. Iodice, I. Rendina, P.M. Sarro: IEEE Photon. Technol. Lett. 7, 363 (1995)

6 G.V. Treyz, P.G. May, J.M. Halbout: Appl. Phys. Lett. 59, 771 (1991)

7 C.Z. Zhao, G.Z. Li, E.K. Liu, Y. Gao, X.D. Liu: Appl. Phys. Lett. 67, $2448(1995)$

8 L.B. Soldano and E.C.M. Pennings: J. Lightwave Technol. 13, 615 (1995)

9 P. Dainesi, A. Küng, M. Chabloz, A. Lagos, Ph. Flückiger, A. Ionescu, P. Fazan, M. Declerq, Ph. Renaud, Ph. Robert: Photon. Technol. Lett. 12, 660 (2000)

10 R.A. Soref, J. Schmidtchen, K. Petermann: IEEE J. Quantum Electron. QE-27, 1971 (1991)

11 C. De Bernardi, A. Küng, O. Leminger: In Photonic Devices for Telecommunications, ed. by G. Guekos (Springer-Verlag, Berlin, Heidelberg 1999) pp. 112-114

12 J.P. Colinge: Silicon-on-Insulator Technology: Materials to VLSI (Kluwer 1991) pp. 38-48 